\title{
Elliptic curve Cryptography and Diffie- Hellman Key exchange
}

\author{
Dr.S.Vasundhara \\ Asst. Prof of Mathematics G.Narayanamma Institute of Technology \& science(For Women) \\ Shaikpet, Hyderabad. Telengana
}

Abstract: In this paper an introduction of Elliptic curve cryptography explained Then the Diffie- Hellman algorithm was explained with clear examples.

Keywords: Cryptography Elliptic curve cryptography, Diffie-Hellman Key exchange.

\section{Introduction}

The history of cryptography is long and interesting. It has a very considerable turning point when two researchers from Stanford, Whitfield Diffie and Martin Hellman, published the paper "New Directions in Cryptography" in 1976. They preface the new idea of public key cryptography in the paper.

Public-key cryptography and [4]symmetric-key cryptography are two main categories of cryptography. The Well-known public-key cryptography algorithms are RSA (Rivest, et al. 1978), El-Gamal and Elliptic Curve Cryptography. Presently, there are only three problems of public key cryptosystems that are considered to be both secure and effective (Certicom, 2001). Table 1.1 shows these mathematical problems and the cryptosystems that rely on such problems.

\begin{tabular}{|l|l|l|l|}
\hline & Mathematical problem & Detail & Cryptosystem \\
\hline 1 & $\begin{array}{l}\text { Integer Factorization problem } \\
\text { (IFP) }\end{array}$ & $\begin{array}{l}\text { Given an integer n find its prime } \\
\text { factorization }\end{array}$ & RSA \\
\hline 2 & $\begin{array}{l}\text { Discrete } \\
\text { problem(DLS) }\end{array}$ & $\begin{array}{l}\text { Given integer g and h find x' } \\
\text { such that =g'mod n }\end{array}$ & $\begin{array}{l}\text { Diffie- } \\
\text { Hellman(DH) }\end{array}$ \\
\hline 3 & $\begin{array}{l}\text { Elliptic curve discrete } \\
\text { logarithmic problem(ECDLP) }\end{array}$ & $\begin{array}{l}\text { Given points P and Q on the } \\
\text { curve find 'x' such that Q=xP }\end{array}$ & $\begin{array}{l}\text { Diffie- } \\
\text { Hellman(DH) }\end{array}$ \\
\hline
\end{tabular}

Providing an equivalent level of security with smaller key size is an advantage of ECC compared to RSA. It is very efficient to[1] implement ECC.ECC obtains lower power consumption, and faster computation. It also gains small memory and bandwidth because of its key size length (Dormale, Bulens and Quisquater 2004), (Huang 2007). Such attributes are mainly fascinating in security applications in which calculative power and integrated circuit space are limited. Wireless devices and smart cards present a good example for the constrained devices with limited resources. Cryptography companies such as Certicom Corporation have already implemented ECC in their products for some commercial purposes which are RFID and Zigbee. This company has an agreement with NSA on a set of cryptographic algorithms called suite B. This suite uses Elliptic curves and works over the prime field.

A modular arithmetic performs a main role in public key cryptographic systems (Dormale, et al. 2004). Some of these PKC are the Diffie-Hellman keys exchange algorithm (Diffie and Hellman 1976), the decipherment operation in the RSA algorithm (Quisquater and Couvreur 1982), the US Government Digital Signature Standard (FIPS 2000), and also elliptic curve cryptography (Koblitz 1987).

\section{Diffie-Hellman:}

Diffie-Hellman key exchange (D-H)[11] is a specific method of exchanging cryptographic keys. It is one of the earliest practical examples of key exchange implemented within the field of cryptography. The Diffie-Hellman key exchange method allows two parties that have no prior knowledge of each other to jointly establish a shared secret key over an insecure communications channel. This key can then be used to encrypt subsequent communications using a symmetric key cipher.

The scheme was first published by Whitfield Diffie and Martin Hellman in 1976, although it had been separately invented a few years earlier within GCHQ, the British signals intelligence agency, by Malcolm J. Williamson but was kept classified. In 2002, Hellman suggested the algorithm be called Diffie-Hellman-Merkle key exchange in recognition of Ralph Merkle's contribution to the invention of public-key cryptography (Hellman, 2002).

Although Diffie-Hellman key agreement itself is an anonymous (non-authenticated) key-agreement protocol, it provides the basis for a variety of authenticated protocols, and is used to provide perfect forward 
secrecy in Transport Layer Security's ephemeral modes (referred to as EDH or DHE depending on the cipher suite).

The method was followed shortly afterwards by RSA, an implementation of public key cryptography using asymmetric algorithms.

\section{In 2002, Martin Hellman wrote:}

The system...has since become known as Diffie-Hellman key exchange. While that system was first described in a paper by Diffie and me, it is a public key distribution system, a concept developed by Merkle, and hence should be called 'Diffie-Hellman-Merkle key exchange' if names are to be associated with it. I hope this small pulpit might help in that endeavor to recognize Merkle's equal contribution to the invention of public key cryptography. [1]U.S. Patent 4,200,770, now expired, describes the algorithm and credits Hellman, Diffie, and Merkle as inventors.

\section{Description}

Diffie-Hellman establishes a shared secret[7] that can be used for secret communications by exchanging data over a public network. The following diagram illustrates the general idea of the key exchange by using colors instead of a very large number. The key part of the process is that Alice and Bob exchange their secret colors in a mix only. Finally this generates an identical key that is mathematically difficult (impossible for modern supercomputers to do in a reasonable amount of time) to reverse for another party that might have been listening in on them. Alice and Bob now use this common secret to encrypt and decrypt their sent and received data. Note that the yellow paint is already agreed by Alice and Bob.
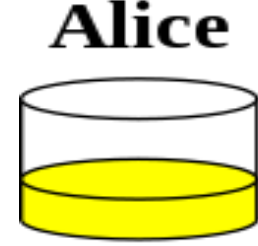

$+$

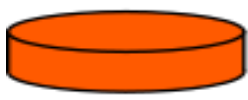

$=$

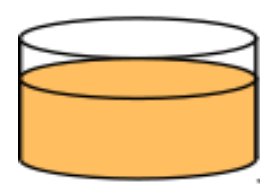

\section{Bob}

Common paint
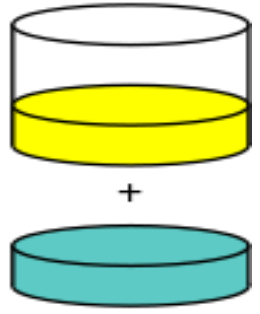

$=$

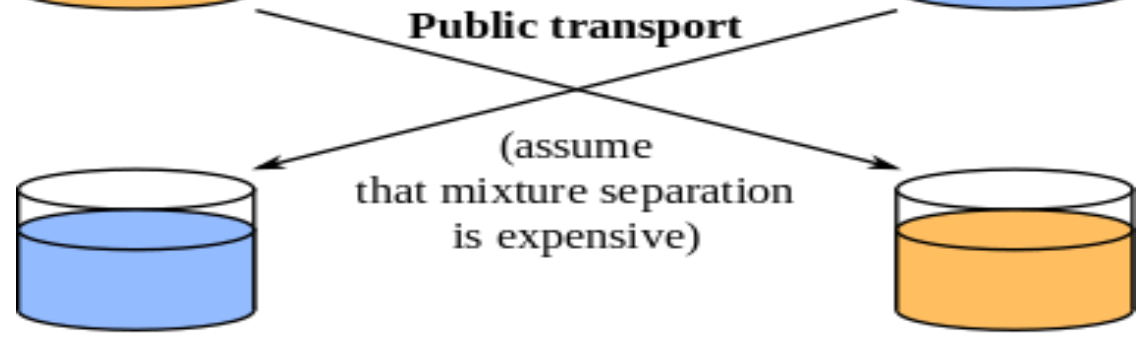

$+$

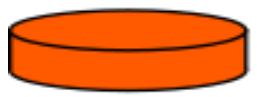

Secret colours

$=$

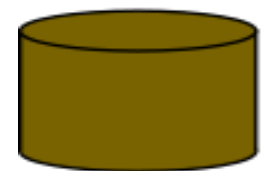

Common secret
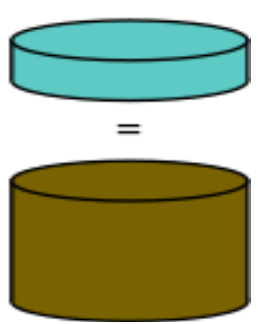

Here is an explanation which includes the encryption's mathematics:

The simplest, and original, implementation of the protocol uses the multiplicative group of integers modulo p, where $\mathrm{p}$ is prime and $\mathrm{g}$ is primitive root $\bmod \mathrm{p}$. Here is an example of the protocol, with non-secret values in blue, and secret values in boldface red: 
Table: 1.1

\begin{tabular}{|l|l|l|l|l|l|l|}
\hline \multicolumn{2}{|l|}{ Alice } & \multicolumn{3}{l|}{ Bob } \\
\hline Secret & Public & Calculates & Sends & Calculates & Public & Secret \\
\hline $\mathrm{a}$ & $\mathrm{p}, \mathrm{g}$ & & $\mathrm{p}, \mathrm{g} \rightarrow$ & & & $\mathrm{b}$ \\
\hline $\mathrm{a}$ & $\mathrm{p}, \mathrm{g}, \mathrm{A}$ & $\mathrm{g}^{\mathrm{a}} \bmod \mathrm{p}=\mathrm{A}$ & $\mathrm{A} \rightarrow$ & & $\mathrm{p}, \mathrm{g}$ & $\mathrm{b}$ \\
\hline $\mathrm{a}$ & $\mathrm{p}, \mathrm{g}, \mathrm{A}$ & & $\leftarrow \mathrm{B}$ & $\mathrm{g}^{\mathrm{b}} \bmod \mathrm{p}=\mathrm{B}$ & $\mathrm{p}, \mathrm{g}, \mathrm{A}, \mathrm{B}$ & $\mathrm{b}$ \\
\hline $\mathrm{a}, \mathrm{s}$ & $\mathrm{p}, \mathrm{g}, \mathrm{A}, \mathrm{B}$ & $\mathrm{B}^{\mathrm{a}} \bmod \mathrm{p}=\mathrm{s}$ & & $\mathrm{A}^{\mathrm{b}} \bmod \mathrm{p}=\mathrm{s}$ & $\mathrm{p}, \mathrm{g}, \mathrm{A}, \mathrm{B}$ & $\mathrm{b}, \mathrm{s}$ \\
\hline
\end{tabular}

1.Alice and Bob agree to use a prime number $p=23$ and base $g=5$.

1. Alice chooses a secret integer $\boldsymbol{a}=\mathbf{6}$, then sends Bob $\mathrm{A}=g^{a} \bmod p$

- $A=5^{6} \bmod 23$

- $\mathrm{A}=\mathbf{1 5 , 6 2 5 \operatorname { m o d } 2 3}$

- $\mathrm{A}=8$

2. Bob chooses a secret integer $\boldsymbol{b}=\mathbf{1 5}$, then sends Alice $\mathrm{B}=g^{\boldsymbol{b}} \bmod p$

- $\mathrm{B}=5^{15} \bmod 23$

- $\mathrm{B}=\mathbf{3 0 , 5 1 7 , 5 7 8 , 1 2 5 \operatorname { m o d } 2 3}$

- $\mathrm{B}=19$

4, Alice computes $\mathbf{s}=B^{a} \bmod p$

- $\mathbf{s}=19^{6} \bmod 23$

- $\mathbf{s}=\mathbf{4 7 , 0 4 5 , 8 8 1 \operatorname { m o d } 2 3}$

- $\mathbf{s}=\mathbf{2}$

5. Bob computes $\mathbf{s}=A^{\boldsymbol{b}} \bmod p$

- $\mathbf{s}=8^{15} \bmod 23$

- $\quad \mathbf{S}=\mathbf{3 5}, \mathbf{1 8 4 , 3 7 2 , 0 8 8 , 8 3 2} \bmod 23$

- $\mathbf{s}=\mathbf{2}$

3. Alice and Bob now share a secret: $\mathbf{s}=\mathbf{2}$. This is because $6^{*} 15$ is the same as $15^{*} 6$. So somebody who had known both these private integers might also have calculated s as follows:

- $\quad s=5^{6^{* 15}} \bmod 23$

- $\mathrm{s}=5^{15^{*}} \bmod 23$

- $\mathbf{s}=5^{90} \bmod 23$

- $\quad \mathrm{S}=\mathbf{8 0 7 , 7 9 3 , 5 6 6 , 9 4 6 , 3 1 6 , 0 8 8 , 7 4 1 , 6 1 0 , 0 5 0 , 8 4 9 , 5 7 3 , 0 9 9 , 1 8 5 , 3 6 3 , 3 8 9 , 5 5 1 , 6 3 9 , 5 5 6 , 8 8 4 , 7 6 5 , 6 2 5} \bmod 23$

- $\mathrm{s}=\mathbf{2}$

Both Alice and Bob have arrived at the same value, because $\left(\mathrm{g}^{\mathrm{a}}\right)^{\mathrm{b}}$ and $\left(\mathrm{g}^{\mathrm{b}}\right)^{\mathrm{a}}$ are equal mod $\mathrm{p}$. Note that only $\mathrm{a}, \mathrm{b}$ and $\mathrm{g}^{\mathrm{ab}}=\mathrm{g}^{\mathrm{ba}}$ mod $\mathrm{p}$ are kept secret. All the other values $-\mathrm{p}, \mathrm{g}, \mathrm{g}^{\mathrm{a}} \bmod \mathrm{p}$, and $\mathrm{g}^{\mathrm{b}} \bmod \mathrm{p}-$ are sent in the clear. Once Alice and Bob compute the shared secret they can use it as an encryption key, known only to them, for sending messages across the same open communications channel. Of course, much larger values of $a, b$, and $\mathrm{p}$ would be needed to make this example secure, since it is easy to try all the possible values of $\mathrm{g}^{\mathrm{ab}} \bmod 23$. There are only 23 possible integers as the result of mod 23. If $\mathrm{p}$ were a prime of at least 300 digits, and $\mathrm{a}$ and $\mathrm{b}$ were at least 100 digits long, then even the best algorithms known today could not find a given only $\mathrm{g}, \mathrm{p}, \mathrm{g}^{\mathrm{b}} \bmod \mathrm{p}$ and $\mathrm{g}^{\mathrm{a}} \bmod \mathrm{p}$, even using all of mankind's computing power. The problem is known as the discrete logarithm problem. Note that $g$ need not be large at all, and in practice is usually either 2,3 or 5.

\section{Here's a more general description of the protocol:}

Alice and Bob agree on a finite cyclic group $G$ and a generating element $g$ in $G$. (This is usually done long before the rest of the protocol; $g$ is assumed to be known by all attackers.) We will

Write the group $G$ multiplicatively.

Alice picks a random natural number $a$ and sends $g^{a}$ to Bob.

Bob picks a random natural number $b$ and sends $g^{b}$ to Alice.

Alice computes $\left(g^{b}\right)^{a}$.

Bob computes $\left(g^{a}\right)^{b}$.

Both Alice and Bob are now in possession of the group element $\mathrm{g}^{\mathrm{ab}}$, which can serve as the shared secret key. The values of $\left(\mathrm{g}^{\mathrm{b}}\right)^{\mathrm{a}}$ and $\left(\mathrm{g}^{\mathrm{a}}\right)^{\mathrm{b}}$ are the same because groups are power associative. (See also exponentiation.)

In order to decrypt a message $\mathrm{m}$, sent as $\mathrm{mg}^{\mathrm{ab}}$, Bob (or Alice) must first compute $\left(\mathrm{g}^{\mathrm{ab}}\right)^{-1}$, as follows:

Bob knows $|\mathrm{G}|, \mathrm{b}$, and $\mathrm{g}^{\mathrm{a}}$. A result from group theory establishes that from the construction of $\mathrm{G}, \mathrm{x}^{|\mathrm{G}|}=1$ for all $\mathrm{x}$ in $\mathrm{G}$.

Bob then calculates $\left(\mathrm{g}^{\mathrm{a}}\right)^{|\mathrm{G}|-\mathrm{b}}=\mathrm{g}^{\mathrm{a}(|\mathrm{G}|-\mathrm{b})}=\mathrm{g}^{\mathrm{a}|\mathrm{G}|-\mathrm{ab}}=\mathrm{g}^{\mathrm{a}|\mathrm{G}|} \mathrm{g}^{-\mathrm{ab}}=\left(\mathrm{g}^{|\mathrm{G}|}\right)^{\mathrm{a}} \mathrm{g}^{-\mathrm{ab}}=1^{\mathrm{a}} \mathrm{g}^{-\mathrm{ab}}=\mathrm{g}^{-\mathrm{ab}}=\left(\mathrm{g}^{\mathrm{ab}}\right)^{-1}$.

DOI: $10.9790 / 5728-1301015661 \quad$ www.iosrjournals.org $\quad 58 \mid$ Page


When Alice sends Bob the encrypted message, $\mathrm{mg}^{\mathrm{ab}}$, Bob applies $\left(\mathrm{g}^{\mathrm{ab}}\right)^{-1}$ and recovers $\mathrm{mg}^{\mathrm{ab}}\left(\mathrm{g}^{\mathrm{ab}}\right)^{-1}=\mathrm{m}(1)=\mathrm{m}$.

Here is a chart to help simplify who knows what. (Eve is an eavesdropper-she watches what is sent between Alice and Bob, but she does not alter the contents of their communications.)

Let $\mathbf{s}=$ shared secret key. $\mathbf{s}=\mathbf{2}$

Let $\mathrm{g}=$ public base. $\mathrm{g}=5$

Let $\mathrm{p}=$ public (prime) number. $\mathrm{p}=23$

Let $\mathbf{a}=$ Alice's private key. $\mathbf{a}=\mathbf{6}$

Let $\mathrm{A}=$ Alice's public key. $\mathrm{A}=\mathrm{g}^{\mathrm{a}} \bmod \mathrm{p}=8$

Let $\mathbf{b}=$ Bob's private key. $\mathbf{b}=\mathbf{1 5}$

Let $\mathrm{B}=$ Bob's public key. $\mathrm{B}=\mathrm{g}^{\mathbf{b}} \bmod \mathrm{p}=19$

Table:1.2

\begin{tabular}{|c|c|c|c|c|c|}
\hline \multicolumn{2}{|l|}{ Alice } & \multicolumn{2}{|l|}{ Bob } & \multicolumn{2}{|l|}{ Eve } \\
\hline Knows & $\begin{array}{l}\text { doesn't } \\
\text { know }\end{array}$ & Knows & $\begin{array}{l}\text { doesn't } \\
\text { know }\end{array}$ & knows & $\begin{array}{l}\text { doesn't } \\
\text { know }\end{array}$ \\
\hline $\mathrm{p}=23$ & $\mathbf{b}=?$ & $\mathrm{p}=23$ & $a=?$ & $\mathrm{p}=23$ & $a=?$ \\
\hline base $g=5$ & & base $g=5$ & & base $g=5$ & $\mathrm{~b}=?$ \\
\hline$a=6$ & & $b=15$ & & & $\mathrm{~S}=?$ \\
\hline$A=5^{6} \bmod 23=8$ & & $\mathrm{~B}=5^{15} \bmod 23=19$ & & $A=5^{a} \bmod 23=8$ & \\
\hline $\mathrm{B}=5^{\mathrm{b}} \bmod 23=19$ & & $A=5^{a} \bmod 23=8$ & & $\mathrm{~B}=5^{\mathrm{b}} \bmod 23=19$ & \\
\hline $\mathrm{s}=19^{6} \bmod 23=2$ & & $\mathrm{~s}=8^{15} \bmod 23=2$ & & $\mathrm{~s}=19^{\mathrm{a}} \bmod 23$ & \\
\hline $\mathrm{s}=8^{\mathrm{b}} \bmod 23=2$ & & $\mathrm{~s}=19^{\mathrm{a}} \bmod 23=2$ & & $\mathrm{~s}=8^{\mathrm{b}} \bmod 23$ & \\
\hline $\begin{array}{l}\mathrm{s}=19^{6} \bmod 23=8^{\mathrm{b}} \bmod \\
23\end{array}$ & & $\begin{array}{l}\mathrm{s}=8^{15} \bmod 23=19^{\mathrm{a}} \\
\bmod 23\end{array}$ & & $\begin{array}{l}\mathrm{s}=19^{\mathrm{a}} \bmod 23=8^{\mathrm{b}} \\
\bmod 23\end{array}$ & \\
\hline$S=2$ & & $S=2$ & & & \\
\hline
\end{tabular}

Note: It should be difficult for Alice to solve for Bob's private key or for Bob to solve for Alice's private key. If it is not difficult for Alice to solve for Bob's private key (or vice versa), Eve may simply substitute her own private / public key pair, plug Bob's public key into her private key, produce a fake shared secret key, and solve for Bob's private key (and use that to solve for the shared secret key. Eve may attempt to choose a public / private key pair that will make it easy

for her to solve for Bob's private key). A demonstration of Diffie-Hellman (using numbers too small for practical use) is given here.

operation with more than two parties

Diffie-Hellman key agreement is not limited to negotiating a key shared by only two participants. Any number of users can take part in an agreement by performing iterations of the agreement protocol and exchanging intermediate data (which does not itself need to be kept secret). For example, Alice, Bob, and Carol could participate in a Diffie-Hellman agreement as follows, with all operations taken to be modulo $p$ :

The parties agree on the algorithm parameters $p$ and $g$.

The parties generate their private keys, named $a, b$, and $c$.

Alice computes $g^{a}$ and sends it to Bob.

Bob computes $\left(g^{a}\right)^{b}=g^{a b}$ and sends it to Carol.

Carol computes $\left(g^{a b}\right)^{c}=g^{a b c}$ and uses it as her secret.

Bob computes $g^{b}$ and sends it to Carol.

Carol computes $\left(g^{b}\right)^{c}=g^{b c}$ and sends it to Alice.

Alice computes $\left(g^{b c}\right)^{a}=g^{b c a}=g^{a b c}$ and uses it as her secret.

Carol computes $g^{c}$ and sends it to Alice.

Alice computes $\left(g^{c}\right)^{a}=g^{c a}$ and sends it to Bob. 
Bob computes $\left(g^{c a}\right)^{b}=g^{c a b}=g^{a b c}$ and uses it as his secret.

An eavesdropper has been able to see $g^{a}, g^{b}, g^{c}, g^{a b}, g^{a c}$, and $g^{b c}$, but cannot use any

Combination of these to reproduce $g^{a b c}$.

To extend this mechanism to larger groups, two basic principles must be followed:

Starting with an "empty" key consisting only of $g$, the secret is made by raising the current value to every participant's private exponent once, in any order (the first such exponentiation yields the participant's own public key).

Any intermediate value (having up to $N-1$ exponents applied, where $N$ is the number of participants in the group) may be revealed publicly, but the final value (having had all $N$ exponents applied) constitutes the shared secret and hence must never be revealed publicly. Thus, each user must obtain their copy of the secret by applying their own private key last (otherwise there would be no way for the last contributor to communicate the final key to its recipient, as that last contributor would have turned the key into the very secret the group wished to protect).

These principles leave open various options for choosing in which order participants contribute to keys. The simplest and most obvious solution is to arrange the $N$ participants in a circle and have $N$ keys rotate around the circle, until eventually every key has been contributed to by all $N$ participants (ending with its owner) and each participant has contributed to $N$ keys (ending with their own). However, this requires that every participant perform $N$ modular exponentiations.

By choosing a more optimal order, and relying on the fact that keys can be duplicated, it is possible to reduce the number of modular exponentiations performed by each participant to $\log _{2}(N)+1_{\text {using a divide-and- }}$ conquer-style approach, given here for eight participants:

1. Participants A, B, C, and D each perform one exponentiation, yielding $g^{a b c d}$; this value is sent to E, F, G, and $\mathrm{H}$. In return, participants A, B, C, and D receive $g^{\text {efgh }}$.

2. Participants A and B each perform one exponentiation, yielding $g^{\text {efghab }}$, which they send to $\mathrm{C}$ and D, while C and D do the same, yielding $g^{\text {efghcd }}$, which they send to A and B.

3. Participant A performs an exponentiation, yielding $g^{\text {efghcda }}$, which it sends to B; similarly, B sends $g^{e f g h c d b}$ to A. C and D do similarly.

4. Participant A performs one final exponentiation, yielding the secret $g^{\text {efghcdba }}=g^{a b c d e f g h}$, while B does the same to get $g^{\text {efghcdab }}=g^{\text {abcdefgh }}$; again, C and D do similarly.

5. Participants E through $\mathrm{H}$ simultaneously perform the same operations using $g^{a b c d}$ as their starting point.

Upon completing this algorithm, all participants will possess the secret $g^{a b c d e f g h}$, but each participant will have performed only four modular exponentiations, rather than the eight implied by a simple circular arrangement.

Security: The protocol is considered secure against eavesdroppers if $G$ and $g$ are chosen properly. The eavesdropper ("Eve") would have to solve the Diffie-Hellman problem to obtain $g^{a b}$. This is currently considered difficult. An efficient algorithm to solve the discrete logarithm problem would make it easy to compute $a$ or $b$ and solve the Diffie-Hellman problem, making this and many other public key cryptosystems insecure.

The order of $G$ should be prime or have a large prime factor to prevent use of the Pohlig-Hellman algorithm to obtain $a$ or $b$. For this reason, a Sophie Germain prime $q$ is sometimes used to calculate $p=2 q+1$, called a safe prime, since the order of $G$ is then only divisible by 2 and $q . g$ is then sometimes chosen to generate the order $q$ subgroup of $G$, rather than $G$, so If Alice and Bob use random number generators whose outputs are not completely random and can be predicted to some extent, then Eve's task is much easier.

The secret integers $a$ and $b$ are discarded at the end of the session. Therefore, Diffie-Hellman key exchange by itself trivially achieves perfect forward secrecy because no long-term private keying material exists to be disclosed.

In the original description, the Diffie-Hellman exchange by itself does not provide authentication of the communicating parties and is thus vulnerable to a man-in-the-middle attack. A person in the middle may establish two distinct Diffie-Hellman key exchanges, one with Alice and the other with Bob, effectively 
masquerading as Alice to Bob, and vice versa, allowing the attacker to decrypt (and read or store) then reencrypt the messages passed between them. A method to authenticate the communicating parties to each other is generally needed to prevent this type of attack. Variants of Diffie-Hellman, such as STS, may be used instead to avoid these types of attacks.

Other uses

\section{Password-authenticated key agreement}

When Alice and Bob share a password, they may use a password-authenticated key agreement (PAKE) form of Diffie-Hellman to prevent man-in-the-middle attacks. One simple scheme is to make the generator $g$ the password. A feature of these schemes is that an attacker can only test one specific password on each iteration with the other party, and so the system provides good security with relatively weak passwords. This approach is described in

ITU-T Recommendation X.1035, which is used by the G.hn home networking standard.

Public Key:

It is also possible to use Diffie-Hellman as part of a public key infrastructure. Alice's public key is simply $\left(g^{a} \bmod p, g, p\right)$. To send her a message Bob chooses a random $b$, and then sends Alice $g^{b} \bmod p$ (un-encrypted) together with the message encrypted with symmetric key $\left(g^{a}\right)^{b} \bmod p$. Only Alice can decrypt the message because only she has $a$. A preshared public key also prevents man-in-themiddle attacks.

In practice, Diffie-Hellman is not used in this way, with RSA being the dominant public key algorithm. This is largely for historical and commercial reasons, namely that RSA created a Certificate Authority that became Verisign. Diffie-Hellman cannot be used to sign certificates, although the ElGamal and DSA signature algorithms are related to it. However, it is related to MQV, STS and the IKE component of the IP sec protocol suite for securing Internet Protocol communications.

\section{References}

[1]. Cole, Eric, Jason Fossen, Stephen Northcutt, Hal Pomeranz. SANS Security Essentials with CISSP CBK, Version 2.1.USA: SANS Press, 2003

[2]. J.Edge,an introduction to elliptic curve ,cryptography, http://Iwn.net/Articles/174127/.2006

[3]. N.Koblitz,A course in Number theory and cryptography, $2^{\text {nd }}$ ed.,brookes/Cole, 1997.

[4]. J.H.Silverman,The Arithmetic of Elliptic curves, Springer -Verlag,1986.

[5]. RSA" Wikipedia.wikipedia,n.d.web.09 feb 2011.Stalings,William. Cryptography and network security.fourth,pearson,2009.print.

[6]. Alfredj Menezes, paul c,vanoorschot and scott A.vanstone,guide to Elliptic curve Cryptography ,1996.

[7]. Diffie, W., and M. E. Hellman. "New directions in cryptography." IEEE Transactions on Information Theory, 1976: 644- 654.

[8]. Stalling, W. Network and Internet work Scurity. IEEE Press, 1995.

[9]. Tata, E. "Elliptic Curve Cryptography, An Implementation Guide.” In Anoop MS. India: Anoop, MS, 2007

[10]. Rivest, R. L., A. Shamir, and L. Adleman. "A Method for Obtaining Digital Signatures and Public-Key Cryptosystems." Communications of the ACM, 1978: 120-126.

[11]. Diffie, W., and M. E. Hellman. "New directions in cryptography." IEEE Transactions on Information Theory, 1976: 644- 654. 